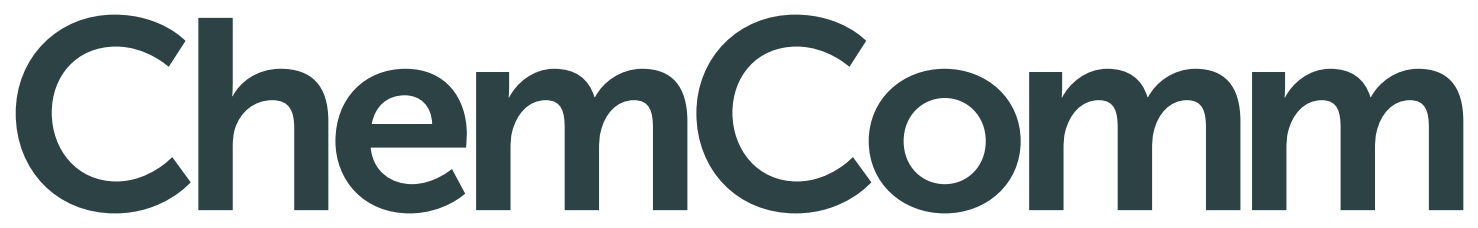

Chemical Communications www.rsc.org/chemcomm

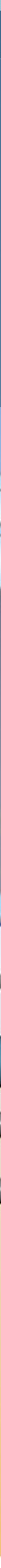

ISSN 1359-7345

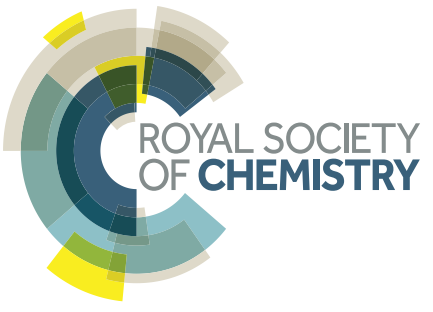




\section{Amphiphilic COSAN and $12-$ COSAN crossing synthetic lipid membranes: planar bilayers and liposomes}

\begin{abstract}
50,6700
Received 18th February 2014 Accepted 24th March 2014
\end{abstract}

Cite this: Chem. Commun., 2014

DOI: $10.1039 / \mathrm{c} 4 \mathrm{cc} 01283 f$

www.rsc.org/chemcomm

\author{
Carmina Verdiá-Báguena, ${ }^{a}$ Antonio Alcaraz, ${ }^{a}$ Vicente M. Aguilella, ${ }^{a}$ Ana M. Cioran, ${ }^{b}$ \\ Shoji Tachikawa, ${ }^{c}$ Hiroyuki Nakamura, ${ }^{d}$ Francesc Teixidor ${ }^{b}$ and Clara Viñas*b
}

The boron-rich cobaltabisdicarbollide (COSAN) and its $8,8^{\prime}-12$ derivative (I2-COSAN), both of purely inorganic nature, are shown to cross through synthetic lipid membranes. These results reveal unexpected properties at the interface of biological and synthetic membranes.

All biological membranes are formed by self-assembly of lipid molecules so that their polar head groups are exposed to the aqueous environment and their fatty acid tails face internally within the membrane, creating a hydrophobic barrier. Membrane integrity is essential for cell regulatory processes, maintaining the osmotic equilibrium that ensures the proper distribution of ions and metabolites, and controlling the biomolecular flow and the electrical signal transduction across membranes. The ability to form membranes is not limited to polar lipid molecules. One example is the inorganic, boron-based molecule cobaltabisdicarbollide, $\left[3,3^{\prime}-\mathrm{Co}\left(1,2-\mathrm{C}_{2} \mathrm{~B}_{9} \mathrm{H}_{11}\right)_{2}\right]^{-}$, commonly known as COSAN (Fig. 1), which comprises of a cobalt atom sandwiched by two carboranyl clusters. ${ }^{1}$ Structurally, COSAN is very different from the lipid molecules that make up biological membranes. The structure exhibits both electrostatic interactions, via a dispersed negative ionic charge covering the whole molecule, ${ }^{2}$ and non-bonding intermolecular interactions between its weakly polarized B-H and C-H bonds. ${ }^{3}$ This duality imparts a molecular property of being simultaneously hydrophobic and hydrophilic, and makes COSAN soluble in both water and oils. The polarized lipid molecules that make up biological membranes also possess amphiphilic properties, and can assemble into membranes and vesicles formed from lipid bilayers. ${ }^{4,5}$ However, unlike lipid bilayer membranes, the membranes of COSAN vesicles are monolayers. ${ }^{4}$ Recently, $\left[3,3^{\prime}-\mathrm{Co}\left(8-\mathrm{I}-1,2-\mathrm{C}_{2} \mathrm{~B}_{9} \mathrm{H}_{10}\right)_{2}\right]^{-}$, I2-COSAN, has also been found to

\footnotetext{
${ }^{a}$ Laboratory of Molecular Biophysics, Dept. of Physics, Universitat Jaume I, 12071 Castelló, Spain

${ }^{b}$ Institut de Ciència de Materials de Barcelona (CSIC), Campus de la U.A.B., E-08193 Bellaterra, Spain. E-mail: clara@icmab.es; Fax: +34 935805729

${ }^{c}$ Department of Life Sciences, Faculty of Science, Gakushuin University, Mejiro, Toshima-ku, Tokyo, 171-8588, Japan

${ }^{d}$ Chemical Resources Laboratory, Tokyo Institute of Technology R1-13,

4259 Nagatsuta-cho, Midori-ku, Yokohama, 226-8503, Japan
}

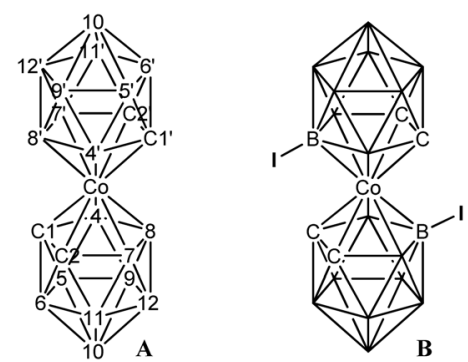

Fig. 1 (A) Vertex numbering of an anionic COSAN cluster. (B) [3, $3^{\prime}-\mathrm{Co}-$ $\left.\left(8-1-1,2-\mathrm{C}_{2} \mathrm{~B}_{9} \mathrm{H}_{10}\right)_{2}\right]^{-}, 12-\mathrm{COSAN}$.

self-assemble into a lyotropic lamellar phase with sufficient curvature to create closed vesicles. ${ }^{5}$

The lamellae formed by I2-COSAN are made of monomolecular sheets such as clay systems, but with the sole difference that they are not covalently bound. The lamella formation presumably originates from intermolecular dihydrogen bonds, $\mathrm{C}-\mathrm{H}^{\delta+\ldots \delta} \mathrm{H}-\mathrm{B} .{ }^{1,5}$ The amphiphilic properties of COSAN and I2-COSAN in water are essential, if they are to have realistic applications in medicine as building blocks for drug delivery or in boron-neutron capture therapy (BNCT). Recently, we demonstrated that COSAN and I2-COSAN accumulate within living cells, where they can be detected by $\nu$ B-H Raman microspectroscopy. ${ }^{6}$ In this communication, the ability of COSAN and I2-COSAN to transfer across synthetic and liposome membranes has been studied for the first time. The COSAN and I2-COSAN transit is achieved without disrupting membrane integrity, and this process occurs with zero-order kinetics against the apparent concentration gradient.

We began this work by investigating the interaction of COSAN with cell-free artificial lipid bilayer membranes. The membranes were formed by monolayer apposition using the Montal-Mueller technique, and analysed using planar lipid bilayer electrophysiology. Monolayers were prepared from a solution of $1 \%$ pure 1,2-dioleoylsn-glycero-3-phosphocholine (DOPC) in pentane. Lipid was added on 70-90 $\mu \mathrm{m}$ diameter orifices in the $15 \mu \mathrm{m}$-thick Teflon partition that separated two identical chambers ${ }^{8}$ with aqueous solutions of $10 \mu \mathrm{M}$ $\mathrm{NaCl}$ at pH 6.0. An Axopatch $200 \mathrm{~B}$ amplifier was used to measure the 


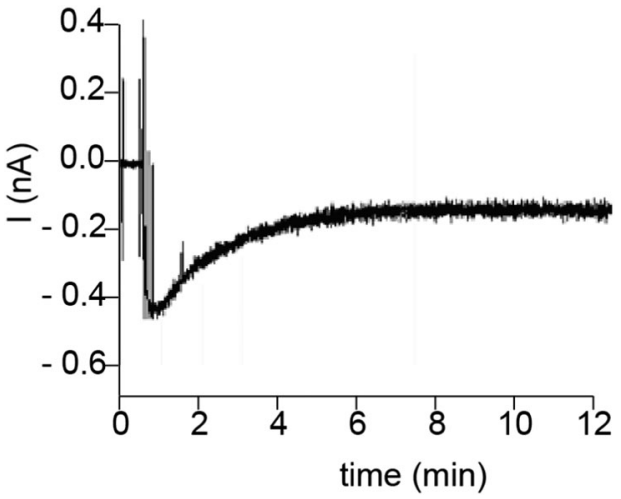

Fig. 2 A typical current recording against time obtained when COSAN is applied to one side of a planar bilayer formed from DOPC. Current initiates as a strong, but transient, depolarization before stabilizing as a continuous, steady negative current across the membrane.

current registered after COSAN addition in the feeding side of the chamber. The COSAN concentration in the stripping side was calculated from the boron concentration determined by ICP-MS. ICP-MS measurements were performed using a quadrupole mass spectrometer Agilent $7500 \mathrm{cx}$ with a collision cell (Agilent technologies, USA).

We found that application of either the protonated form $(\mathrm{H}[\mathrm{COSAN}])$ or the sodium salt $(\mathrm{Na}[\mathrm{COSAN}])$ to the feeding side of the artificial lipid membrane resulted in initial current depolarization, which then stabilized after 5 minutes as a steady negative ionic current across the membrane (Fig. 2). This occurred without application of a trans-membrane voltage, indicating that COSAN directly translocates across the membrane without a driving force. Membrane electrical capacitance remained constant throughout each experiment, indicating that the lipid bilayer remained intact and no aqueous pores were formed in the membrane. Lipid composition does not influence the COSAN transport rate, as no significant permeation differences were seen for model membranes of neutral lipid composition that mimic either prokaryotic (DPhPC: 1,2-diphytanoyl-sn-glycero-3-phosphocholine) or eukaryotic (1,2-dioleoyl-sn-glycero-3-phosphocholine, DOPC) cell membranes. Electrostatic properties of the membrane have small but still measurable effects. A slight reduction of the COSAN transport rate was observed when the negatively charged membranes DOPC/DOPS $(4: 1)$, which mimic the surface charge density of a typical plasma membrane, were used.

Next, the electrophysiology of COSAN and I2-COSAN permeating through planar membranes was studied. The translocation of $\mathrm{Na}[\mathrm{I} 2-\mathrm{COSAN}]$ is lower compared with that of $\mathrm{H}[\mathrm{COSAN}]$ and $\mathrm{Na}[\mathrm{COSAN}]$, however, in all cases the permeation rate was independent of the initial concentration, showing zero order kinetics. This indicates that the rate-determining step is not the diffusion across the membrane but the partitioning of COSAN or I2-COSAN into the lipid phase. ${ }^{9}$ Interestingly, different permeation rates were found for $\mathrm{H}[\mathrm{COSAN}]$ and $\mathrm{Na}[\mathrm{COSAN}]$ (Fig. 3). The reason for this is still unclear. Previous studies showed that the presence of sodium ions drastically modifies the COSAN aggregation process. ${ }^{4 a}$ In fact, it was observed that sodium ions favour larger nano-vesicles (diameter from 80 to $890 \mathrm{~nm}$ ) than protons do (diameter nearly

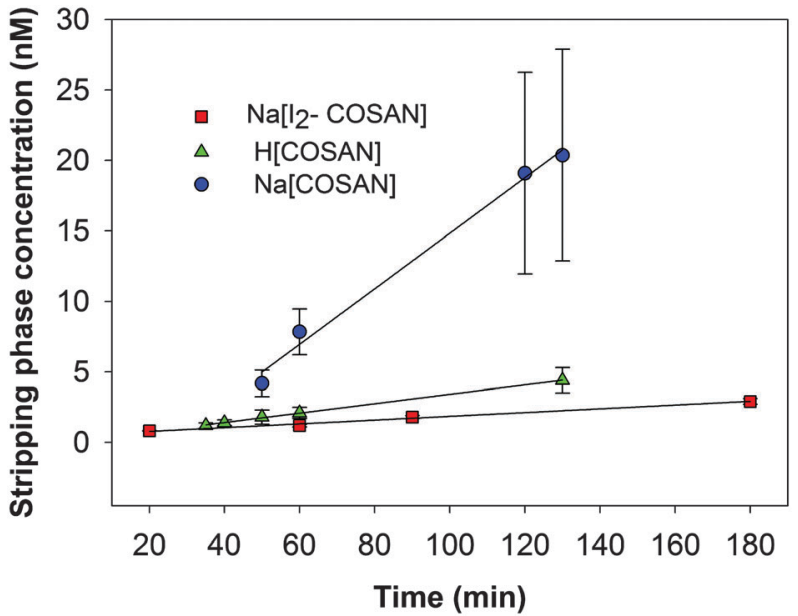

Fig. 3 Permeation rate of $\mathrm{Na}[\mathrm{COSAN}], \mathrm{H}[\mathrm{COSAN}]$ and Na[I2-COSAN] across neutral planar membranes as measured by ICP-MS. All of them showed zero-order kinetics. Initial concentrations of the three compounds were $100 \mu \mathrm{M}$. Error bars are standard deviations resulting from 10 independent experiments.

$40 \mathrm{~nm}) .{ }^{4 a}$ This suggests that absorption-desorption rates at solutionmembrane interfaces may be determined by the structural form of COSAN molecules.

The direct measurements of COSAN concentrations by inductively coupled plasma mass spectrometry (ICP-MS) in the stripping side matched the estimated concentration calculated from ion current measurements. This close agreement between the two independent estimations indicates that transport of other small ions across the lipid bilayer is minimal, if at all. As an anion, COSAN would not be expected to travel directly through a lipid bilayer due to its high Born energy, ${ }^{10}$ calculated as $\sim 80 \mathrm{~kJ} \mathrm{~mol}^{-1}$, or more than $30 \mathrm{kT}$, for an ideal COSAN anion $(1.1 \mathrm{~nm} \times 0.6 \mathrm{~nm}) .{ }^{4 a}$ We predict that the unusual dual hydrophilic and lipophilic properties of the COSAN structure overcome this energy barrier allowing COSAN ions to cross the lipid membrane. COSAN permeation would occur via the sequence of absorption at the solution-membrane interface, translocation across the lipid phase and desorption at the membranesolution interface.

To directly visualize this transferring behaviour, mixtures of COSAN nano-vesicles ${ }^{12}$ and liposome solutions ${ }^{12}$ were examined using cryo-TEM. ${ }^{11}$ COSAN forms spherical nano-vesicles in water with a radius of approximately $20 \mathrm{~nm}$ and a membrane nearly $1 \mathrm{~nm}$ thick, ${ }^{4 a}$ whereas liposomes form mostly unilamellar bilayer vesicles with a membrane thickness of about $4.5 \mathrm{~nm}$ (Fig. 4). In COSAN : liposome mixtures, $1: 4, \mathrm{v}: \mathrm{v}$ (an equivalent COSAN concentration of $4 \mathrm{mM}$ ), COSAN vesicles fuse with the liposome membrane (Fig. 4a). At a lower liposome ratio $(1: 3, \mathrm{v}: \mathrm{v}$; an equivalent COSAN concentration of $5 \mathrm{mM}$ ), additional intermediate effects are seen (Fig. $4 \mathrm{~b}$ ). Often, two or more liposome units become linked via COSAN nanovesicles. At this interface, COSAN nano-vesicles exhibit a dramatic morphological change to form a planar lamellar microstructure (Fig. 4c). This lamellar form of COSAN appeared to diffuse from the membrane into the liposome center recovering its spherical monolayer vesicle morphology (Fig. 4b, arrows). These liposomeCOSAN double vesicles were larger than the initial liposome 
a

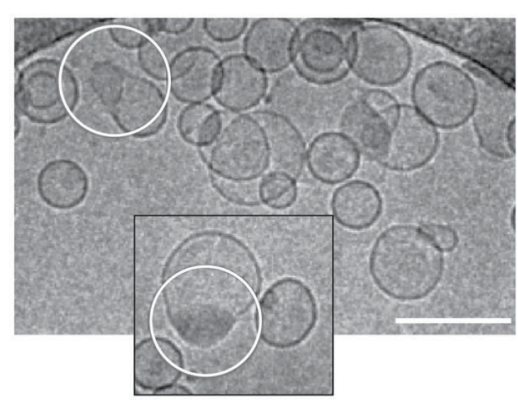

b

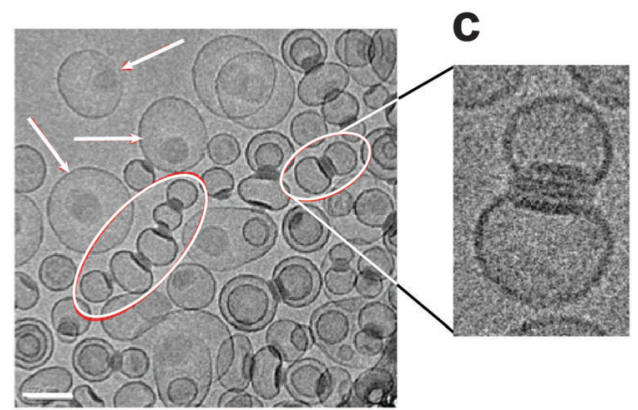

Fig. 4 Visualisation of the interaction between COSAN vesicles and liposomes. (a) CryoTEM image of COSAN : liposomes (1:4) suspended in vitreous ice. Circles highlight fusion between COSAN vesicles and liposomes. Scale bar $200 \mathrm{~nm}$. (b) CryoTEM image of COSAN : liposomes (1:3) suspended in vitreous ice. The circles highlight the joining of two or more liposome units linked by COSAN. Arrows indicate the complete penetration of the COSAN inside the liposome and the recovery of the monolayer vesicle form. Scale bar $200 \mathrm{~nm}$. (c) $50000 \times$ magnification shows the planar multilayer morphology of COSAN at the interface of two liposomes.

population, suggesting that the process of COSAN vesicle encapsulation may cause multiple liposomes to fuse.

The crossing of the encapsulated $\mathrm{H}[\mathrm{COSAN}]$ through planar lipid bilayer electrophysiology membranes was also studied. Samples containing 1:3 COSAN : liposome $(\mathrm{v}: \mathrm{v})$ were prepared with equivalent COSAN concentrations of $10,25,50$ and $100 \mu \mathrm{M}$.

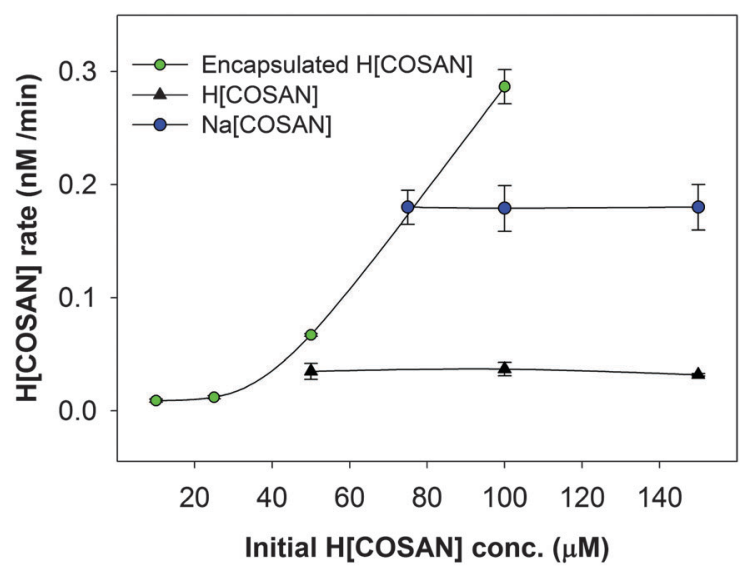

Fig. 5 Translocation of $\mathrm{H}[\mathrm{COSAN}]$ and $\mathrm{Na}[\mathrm{COSAN}]$ through synthetic lipid membranes in their original form and encapsulated into liposomes. The comparison shows two different mechanisms. The permeation rate of encapsulated $\mathrm{H}[\mathrm{COSAN}]$ is strongly dependent on the initial H[COSAN] concentration in the feeding phase, whereas the unencapsulated $\mathrm{H}[\mathrm{COSAN}]$ is not.
As shown in Fig. 5, the encapsulated $\mathrm{H}$ [COSAN] behaves differently from $\mathrm{H}[\mathrm{COSAN}]$. Passive translocation across the planar membrane of the encapsulated COSAN was more efficient than that of plain $\mathrm{H}[\mathrm{COSAN}]$. In addition, the permeation rate of the encapsulated $\mathrm{H}[\mathrm{COSAN}]$ was dependent on the initial concentration, so the accumulated concentration of COSAN in the stripping phase increased exponentially with time. These findings suggest that the rate-determining step for the translocation is the diffusion process across the lipid membrane and not the solution-partitioning seen for plain $\mathrm{H}[\mathrm{COSAN}]$. The absorption and desorption of encapsulated COSAN at solutionmembrane interfaces are much faster than bare COSAN.

All in all, these observations demonstrate that COSAN and I2-COSAN are able to generate vesicles due to their capacity to produce inter-molecular interactions and can transit lipid bilayer membranes without affecting membrane integrity. It has been detected by CryoTEM that two or more liposome units become linked via COSAN nano-vesicles. At this interface, COSAN nano-vesicles exhibit a dramatic morphological change from vesicles to a planar lamellar microstructure that appeared to diffuse from the membrane into the liposome recovering its spherical monolayer vesicle morphology. These results reveal unexpected properties at the interface of biological and synthetic membranes.

This work was supported by Generalitat de Catalunya (2009/ SGR/00279), Generalitat Valenciana (Prometeu 2012/069), Fundació Caixa Castelló-Bancaixa (P1-1B2012-03) and Spanish Ministry of Economy and Competitiveness (CTQ2010-16237 and FIS2010-19810). A.C. thanks MICINN for the FPU grant.

\section{Notes and references}

1 R. N. Grimes, Carboranes, Academic Press, Burlington, MA, 2011.

2 C. Massalles, J. Llop, C. Viñas and F. Teixidor, Adv. Mater., 2002, 14, 826.

3 (a) M. J. Hardie and C. L. Raston, Chem. Commun., 2001, 905; (b) E. J. Juarez-Perez, R. Nuñez, C. Viñas, R. Sillanpää and F. Teixidor, Eur. J. Inorg. Chem., 2010, 2385; (c) C. Viñas, M. Tarrés, P. GonzálezCardoso, P. Farràs, P. Bauduin and F. Teixidor, Dalton Trans., 2014, 5062.

4 (a) P. Bauduin, S. Prevost, P. Farras, F. Teixidor, O. Diat and T. Zemb, Angew. Chem., Int. Ed., 2011, 50, 5298; (b) P. Matějíček, P. Cígler, K. Procházka and V. Král, Langmuir, 2006, 22, 575.

5 D. Brusselle, P. Bauduin, L. Girard, A. Zaulet, C. Viñas, F. Teixidor, I. Ly and O. Diat, Angew. Chem., Int. Ed., 2013, 52, 12114.

6 M. Tarrés, E. Canetta, C. Viñas, F. Teixidor and A. J. Harwood, Chem. Commun., 2014, 50, 3370.

7 M. Montal and P. Mueller, Proc. Natl. Acad. Sci. U. S. A., 1972, 69, 35617; S. M. Bezrukov and I. Vodyanoy, Biophys. J., 1993, 64, 16. 8 V. A. Parsegian, Nature, 1969, 221, 844.

9 N. Lakshminarayanaiah, Equations of membrane biophysics, Academic Press, London, 1984.

10 Vitrified specimens were prepared by placing $3 \mu \mathrm{L}$ of the studied sample suspension on a 400 mesh copper grid with a holey carbon support. Each sample was blotted to a thin film and immediately plunged into liquid ethane in the Leica CPC cryoworkstation. The grids were viewed on a JEOL 2011 transmission electron microscope operating at an accelerating voltage of $200 \mathrm{kV}$. The microscope was equipped with a Gatan cryoholder and the samples were maintained at $-177{ }^{\circ} \mathrm{C}$ during imaging. Electron micrographs were recorded with the Digital Micrograph software package under low electron dose conditions, to minimize electron bean radiation. Images were recorded on a Gatan 794 MSC 600HP cooled charge-coupled device (CCD) camera. 
11 A $2 \mathrm{mM}$ solution of $\mathrm{H}[\mathrm{COSAN}]$ in water. $\mathrm{H}[\mathrm{COSAN}]$ was prepared from the Cs[COSAN] by cationic exchange resin using a wateracetonitrile $(50: 50)$ mixture.

12 Liposomes were prepared from DSPC, DSPE-PEG2000, and cholesterol $(1: 0.11: 1$, molar ratio) according to the reverse-phase evaporation (REV) method. Total lipids of $88 \mathrm{mg}$ were dissolved in $3 \mathrm{~mL}$ of a chloroform-diisopropyl ether mixture $(1: 1, \mathrm{v} / \mathrm{v})$ and $1.5 \mathrm{~mL}$ of distilled water was added to form a w/o emulsion. The emulsion was sonicated for $3 \mathrm{~min}$ and then, the organic solvents were removed under reduced pressure in a rotary evaporator at $60{ }^{\circ} \mathrm{C}$ for $30 \mathrm{~min}$ to obtain a suspension of liposomes. The liposomes obtained were subjected to extrusion 10 times through a polycarbonate membrane filter of $100 \mathrm{~nm}$ pore size (Whatman, 110605, FILTER, 0.1UM, 25MM, Gentaur Molecular Products, Belgium), using an extruder device (LIPEXTM Extruder, Northern Lipids, Canada) thermostated at $60{ }^{\circ} \mathrm{C}$. Purification was accomplished by ultracentrifugation (himac cp $80 \mathrm{wx}$, Hitachi Koki, Japan) at $200000 \mathrm{~g}$ for $60 \mathrm{~min}$ at $4{ }^{\circ} \mathrm{C}$, and the pellets obtained were re-suspended in water $(1.5 \mathrm{~mL})$. 